\title{
Some properties of packing measure with doubling gauge
}

\author{
by \\ Sheng-You Wen (Hubei) and Zhi-Ying Wen (Beijing)
}

\begin{abstract}
Let $g$ be a doubling gauge. We consider the packing measure $\mathcal{P}^{g}$ and the packing premeasure $\mathcal{P}_{0}^{g}$ in a metric space $X$. We first show that if $\mathcal{P}_{0}^{g}(X)$ is finite, then as a function of $X, \mathcal{P}_{0}^{g}$ has a kind of "outer regularity". Then we prove that if $X$ is complete separable, then $\lambda \sup \mathcal{P}_{0}^{g}(F) \leq \mathcal{P}^{g}(B) \leq \sup \mathcal{P}_{0}^{g}(F)$ for every Borel subset $B$ of $X$, where the supremum is taken over all compact subsets of $B$ having finite $\mathcal{P}_{0}^{g}$-premeasure, and $\lambda$ is a positive number depending only on the doubling gauge $g$. As an application, we show that for every doubling gauge function, there is a compact metric space of finite positive packing measure.
\end{abstract}

1. Introduction. Let $g:[0, \infty) \rightarrow[0, \infty)$ be a gauge, i.e., a function which is non-decreasing for $t \geq 0$, right-continuous at $t=0$, and $g(t)=0$ if and only if $t=0$. A gauge $g$ is said to be doubling if there are numbers $c, \delta>0$ such that $g(2 t) \leq c g(t)$ for all $t \in(0, \delta)$. For a doubling gauge $g$ we introduce a non-decreasing function $g_{*}(x)$ as follows:

$$
g_{*}(x)=\liminf _{t \downarrow 0} \frac{g(x t)}{g(t)}, \quad x \in[0, \infty) .
$$

We write $g_{*}(1-0)$ for the left limit of $g_{*}(x)$ at $x=1$.

Let $X$ be a metric space. Let $E \subset X$ and $\delta>0$. A $\delta$-packing of $E$ is defined to be a countable family $\left\{B\left(x_{i}, r_{i}\right)\right\}$ of disjoint closed balls with $2 r_{i} \leq \delta$ and centers $x_{i} \in E$. The packing premeasure of $E$ with respect to the gauge $g$ is defined by

$$
\mathcal{P}_{0}^{g}(E)=\lim _{\delta \rightarrow 0} \mathcal{P}_{\delta}^{g}(E),
$$

where $\mathcal{P}_{\delta}^{g}(E):=\sup \sum g\left(2 r_{i}\right)$, the supremum being taken over all $\delta$-packings

2000 Mathematics Subject Classification: 28A78, 28A80. dition.

Key words and phrases: packing measure, premeasure, gauge function, doubling con-

This research was supported by the Special Funds for Major State Basic Research Projects of China, and partially carried out at the Morningside Center of Mathematics, Chinese Academy of Sciences. 
of $E$. The packing measure of $E$ with respect to the gauge $g$ is defined by

$$
\mathcal{P}^{g}(E)=\inf \left\{\sum_{i=1}^{\infty} \mathcal{P}_{0}^{g}\left(E_{i}\right): E \subset \bigcup_{i=1}^{\infty} E_{i}\right\} .
$$

The packing premeasure and measure with respect to the gauge $t^{s}(s \geq 0)$, which we denote by $\mathcal{P}_{0}^{s}$ and $\mathcal{P}^{s}$ respectively, are the ordinary $s$-dimensional packing premeasure and measure. From the definitions above, $\mathcal{P}_{0}^{g}$ is monotonic and finitely subadditive, and $\mathcal{P}^{g}$ is an outer measure of $\mathcal{P}_{0}^{g}$; for more details, we refer to [3], [8], [9].

Let $K \subset \mathbb{R}^{n}$ be a compact set and $0 \leq s \leq n$. Feng, Hua and Wen [4] proved that if $\mathcal{P}_{0}^{s}(K)<\infty$, then

$$
\mathcal{P}_{0}^{s}(K)=\mathcal{P}^{s}(K) \text {. }
$$

The above conclusion may fail for doubling gauges. M. Csörnyei [1] constructed a compact set $K \subset \mathbb{R}^{1}$ and a doubling gauge $g$ such that

$$
\mathcal{P}^{g}(K)<\mathcal{P}_{0}^{g}(K)<\infty .
$$

Motivated by this fact, we discuss some measure-theoretic properties of the packing measure $\mathcal{P}^{g}$ and the premeasure $\mathcal{P}_{0}^{g}$ with respect to a doubling gauge $g$ in a metric space $X$. It will be shown that if $\mathcal{P}_{0}^{g}(X)<\infty$ then $\mathcal{P}_{0}^{g}$ is "outer regular-like", meaning that

$$
g_{*}(1-0) \inf \mathcal{P}_{0}^{g}(U) \leq \mathcal{P}_{0}^{g}(F) \leq \inf \mathcal{P}_{0}^{g}(U)
$$

for any compact set $F \subset X$, where the infimum is over all open sets containing $F$ (Theorem 1). Furthermore we get a relationship between $\mathcal{P}^{g}$ and $\mathcal{P}_{0}^{g}$ when $X$ is complete separable. Namely, for any Borel set $B \subset X$ we have

$$
\left(g_{*}(1-0)\right)^{2} \sup \mathcal{P}_{0}^{g}(F) \leq \mathcal{P}^{g}(B) \leq \sup \mathcal{P}_{0}^{g}(F),
$$

where the supremum is taken over all compact subsets contained in $B$ with $\mathcal{P}_{0}^{g}(F)<\infty$ (Theorem 2). As a corollary, we show that for every doubling gauge function there is a compact metric space of finite positive packing measure (Theorem 3), which can be regarded as a dual to a result on the Hausdorff measure obtained by A. Dvoretzky [2].

2. The "outer regularity" of a packing premeasure. We start with some statements equivalent to the doubling condition.

Lemma 1. Let $g$ be a gauge. Then the following statements are equivalent:

(a) $g$ is doubling;

(b) $g_{*}(x)>0$ for some $x \in(0,1)$;

(c) $g_{*}(x)>0$ for all $x>0$;

(d) $g_{*}(1-0)>0$. 
Proof. (a) $\Rightarrow$ (b). The doubling condition implies that there is a constant $c \in(0, \infty)$ such that $g(t) \leq c g(t / 2)$ for all $t>0$ small enough, so $g_{*}(1 / 2) \geq$ $1 / c>0$.

(b) $\Rightarrow(\mathrm{c})$. Let $a \in(0,1)$ with $g_{*}(a)>0$. Then $g(a t) \geq \frac{1}{2} g_{*}(a) g(t)$ for $t>0$ small enough. For every $x>0$ choose a positive integer $m$ such that $x \geq a^{m}$. For all $t>0$ small enough we get inductively

$$
g(x t) \geq g\left(a^{m} t\right) \geq \frac{1}{2} g_{*}(a) g\left(a^{m-1} t\right) \geq \cdots \geq\left(\frac{1}{2} g_{*}(a)\right)^{m} g(t),
$$

which yields $g_{*}(x) \geq\left(\frac{1}{2} g_{*}(a)\right)^{m}>0$.

(c) $\Rightarrow(\mathrm{d})$. This is trivial since $g_{*}$ is non-decreasing.

$(d) \Rightarrow(a)$. Since $g_{*}(1-0)>0$, we obtain $g_{*}(x)>0$ for some $x \in(0,1)$. By an argument analogous to $(\mathrm{b}) \Rightarrow(\mathrm{c})$, we get $g_{*}(1 / 2)>0$, which implies that $g$ is doubling.

In the rest of the paper, we assume that $g$ is a doubling gauge. The following theorem shows that if the packing premeasure is finite then it is "outer regular".

Theorem 1. Suppose $X$ is a metric space with $\mathcal{P}_{0}^{g}(X)<\infty$. Then for any compact subset $F$ of $X$,

$$
g_{*}(1-0) A_{F} \leq \mathcal{P}_{0}^{g}(F) \leq A_{F},
$$

where $A_{F}=\inf \left\{\mathcal{P}_{0}^{g}(U): U\right.$ open and $\left.U \supseteq F\right\}$.

Proof. From the monotonicity of $\mathcal{P}_{0}^{g}$, the second inequality of (4) is evident, so we only need to prove the first. Let $\varrho$ denote the metric of $X$. For $\varepsilon>0$ denote by $F_{\varepsilon}$ the open $\varepsilon$-neighborhood of $F$, i.e.

$$
F_{\varepsilon}=\{x \in X: \varrho(x, y)<\varepsilon \text { for some } y \in F\} .
$$

Then $A_{F}=\inf _{\varepsilon>0} \mathcal{P}_{0}^{g}\left(F_{\varepsilon}\right)$ and $0 \leq A_{F}<\infty$ since $\mathcal{P}_{0}^{g}(X)$ is finite. Let $\omega>0$ be arbitrary and choose $\varepsilon>0$ small enough such that

$$
A_{F} \leq \mathcal{P}_{0}^{g}\left(F_{\varepsilon}\right) \leq A_{F}+\omega .
$$

Then, by the definition of $\mathcal{P}_{0}^{g}$, we have

$$
\mathcal{P}_{0}^{g}\left(F_{\varepsilon}\right) \leq \mathcal{P}_{\delta}^{g}\left(F_{\varepsilon}\right) \leq \mathcal{P}_{0}^{g}\left(F_{\varepsilon}\right)+\omega
$$

for $\delta>0$ small enough. Now let $\left\{B\left(x_{i}, r_{i}\right)\right\}_{i=1}^{m}$ be a $\delta$-packing of $F_{\varepsilon}$ such that

$$
\mathcal{P}_{\delta}^{g}\left(F_{\varepsilon}\right)-\omega \leq \sum_{i=1}^{m} g\left(2 r_{i}\right) \leq \mathcal{P}_{\delta}^{g}\left(F_{\varepsilon}\right) .
$$

By the compactness of $F$, we may choose $\left\{y_{i}\right\}_{i=1}^{m} \subset F$ such that

$$
\varrho\left(x_{i}, y_{i}\right)=\varrho\left(x_{i}, F\right), \quad 1 \leq i \leq m .
$$


Let

$$
r_{i}^{*}=\max \left\{r_{i}-\varrho\left(x_{i}, y_{i}\right), 0\right\}, \quad r_{i}^{* *}=\min \left\{\varrho\left(x_{i}, y_{i}\right), r_{i}\right\} .
$$

By the definitions above, we easily see that $r_{i}^{*}+r_{i}^{* *}=r_{i}$. Let $I=\{i$ : $\left.B\left(x_{i}, r_{i}\right) \cap F \neq \emptyset, 1 \leq i \leq m\right\}$. It is obvious that $\left\{B\left(y_{i}, r_{i}^{*}\right)\right\}_{i \in I}$ is a $\delta$-packing of $F$. Thus

$$
P_{\delta}^{g}(F) \geq \sum_{i \in I} g\left(2 r_{i}^{*}\right)=\sum_{i=1}^{m} g\left(2 r_{i}^{*}\right) .
$$

Now let $J=\left\{i: x_{i} \notin F, 1 \leq i \leq m\right\}$ and $1 / 2<t<1$, and choose $0<\varepsilon_{1} \leq 3 \delta$ such that $F_{\varepsilon_{1}} \cap \bigcup_{i \in J} B\left(x_{i}, t r_{i}^{* *}\right)=\emptyset$. We see that if $\left\{B\left(z_{i}, l_{i}\right)\right\}_{i=1}^{\infty}$ is a $3^{-1} \varepsilon_{1}$-packing of $F_{3^{-1} \varepsilon_{1}}$ then $\left\{B\left(z_{i}, l_{i}\right)\right\}_{i=1}^{\infty} \cup\left\{B\left(x_{i}, t r_{i}^{* *}\right)\right\}_{i \in J}$ is a $\delta$-packing of $F_{\varepsilon}$. Thus, in view of $(6)$ and $(7)$, we have

so

$$
\sum_{i=1}^{\infty} g\left(2 l_{i}\right)+\sum_{i \in J} g\left(2 t r_{i}^{* *}\right) \leq A_{F}+2 \omega
$$

$$
\sum_{i \in J} g\left(2 t r_{i}^{* *}\right) \leq 2 \omega
$$

which together with the doubling property of $g$ yields

$$
\sum_{i=1}^{m} g\left(2 r_{i}^{* *}\right)=\sum_{i \in J} g\left(2 r_{i}^{* *}\right) \leq c \sum_{i \in J} g\left(2 t r_{i}^{* *}\right) \leq 2 c \omega,
$$

where $c>0$ is a constant.

Now we are going to estimate the sum on the right hand side of (9). Let

$$
x(\omega)=\omega+2 \sup \left\{x \geq 0: g_{*}(x)<\omega^{1 / 2}\right\} .
$$

From Lemma 1, we have $\lim _{\omega \rightarrow 0} x(\omega)=0$ and $\lim _{\omega \rightarrow 0} \omega / g_{*}(x(\omega))=0$.

Let $I_{\omega}=\left\{i: r_{i}^{* *} \geq r_{i} x(\omega)\right\}$ and $J_{\omega}=\left\{i: r_{i}^{* *}<r_{i} x(\omega)\right\}$. Then, by (10), we have

$$
\sum_{i \in I_{\omega}} g\left(2 r_{i}\right) \leq \sum_{i \in I_{\omega}} \frac{g\left(2 r_{i}^{* *}\right) g\left(2 r_{i}\right)}{g\left(2 r_{i} x(\omega)\right)} \leq 2 c \omega\left(\inf _{0<t \leq \delta} \frac{g(t x(\omega))}{g(t)}\right)^{-1}
$$

which combined with (6), (7), (8) and (11) yields

$$
\begin{aligned}
\sum_{i \in J_{\omega}} g\left(2 r_{i}\right) & =\sum_{i=1}^{m} g\left(2 r_{i}\right)-\sum_{i \in I_{\omega}} g\left(2 r_{i}\right) \\
& \geq A_{F}-\omega-2 c \omega\left(\inf _{0<t \leq \delta} \frac{g(t x(\omega))}{g(t)}\right)^{-1} .
\end{aligned}
$$

From (9) and (12),

$$
\mathcal{P}_{\delta}^{g}(F) \geq \sum_{i \in J_{\omega}} g\left(2 r_{i}^{*}\right) \geq \sum_{i \in J_{\omega}} \frac{g\left(2 r_{i}(1-x(\omega))\right) g\left(2 r_{i}\right)}{g\left(2 r_{i}\right)}
$$




$$
\begin{aligned}
& \geq \inf _{0<t \leq \delta} \frac{g(t(1-x(\omega)))}{g(t)} \sum_{i \in J_{\omega}} g\left(2 r_{i}\right) \\
& \geq \inf _{0<t \leq \delta} \frac{g(t(1-x(\omega)))}{g(t)}\left(A_{F}-\omega-2 c \omega\left(\inf _{0<t \leq \delta} \frac{g(t x(\omega))}{g(t)}\right)^{-1}\right) .
\end{aligned}
$$

Thus by letting $\delta \rightarrow 0$ we get

$$
\mathcal{P}_{0}^{g}(F) \geq g_{*}(1-x(\omega))\left(A_{F}-\omega-\frac{2 c \omega}{g_{*}(x(\omega))}\right) .
$$

Letting $\omega \rightarrow 0$, finally we obtain $\mathcal{P}_{0}^{g}(F) \geq g_{*}(1-0) A_{F}$.

3. The relationship between $\mathcal{P}^{g}$ and $\mathcal{P}_{0}^{g}$. In this section, we will investigate the relation between the packing measure and the premeasure with respect to a doubling gauge in a complete separable metric space.

Lemma 2. Let $X$ be a metric space and let $g$ be a gauge. Then:

(a) for any subset $F$ of $X$, we have

$$
g_{*}(1-0) \mathcal{P}_{0}^{g}(\operatorname{cl}(F)) \leq \mathcal{P}_{0}^{g}(F) \leq \mathcal{P}_{0}^{g}(\operatorname{cl}(F)),
$$

where $\mathrm{cl}(F)$ denotes the closure of $F$;

(b) if $g$ is left-continuous for $t>0$, then $\mathcal{P}_{0}^{g}(F)=\mathcal{P}_{0}^{g}(\operatorname{cl}(F))$.

Proof. (a) To prove (13), it suffices to prove the first inequality. Let $\varepsilon, \delta \in(0,1)$. For every $\delta$-packing $\left\{B\left(x_{i}, r_{i}\right)\right\}$ of $\operatorname{cl}(F)$, choose $\left\{y_{i}\right\} \subset F$ such that $\left\{B\left(y_{i},(1-\varepsilon) r_{i}\right)\right\}$ is a $\delta$-packing of $F$. Then we have

$$
\mathcal{P}_{\delta}^{g}(F) \geq \sum g\left(2(1-\varepsilon) r_{i}\right) \geq \inf _{0<t \leq \delta} \frac{g((1-\varepsilon) t)}{g(t)} \sum g\left(2 r_{i}\right),
$$

so

$$
\mathcal{P}_{\delta}^{g}(F) \geq \inf _{0<t \leq \delta} \frac{g((1-\varepsilon) t)}{g(t)} \mathcal{P}_{\delta}^{g}(\operatorname{cl}(F))
$$

Letting $\delta \rightarrow 0$ and $\varepsilon \rightarrow 0$, we immediately get the desired inequality.

(b) Now assume that $g$ is left-continuous for $t>0$. In this case, from a $\delta$-packing $\left\{B\left(x_{i}, r_{i}\right)\right\}$ of $\operatorname{cl}(F)$ we may construct a $\delta$-packing $\left\{B\left(y_{i}, r_{i}^{*}\right)\right\}$ of $F$ such that for every $i$,

$$
g\left(2 r_{i}^{*}\right) \geq g\left(2 r_{i}\right)-\varepsilon / 2^{i} .
$$

From this we get $\mathcal{P}_{0}^{g}(F) \geq \mathcal{P}_{0}^{g}(\operatorname{cl}(F))$, which yields the required equality immediately.

Lemma 3. Let $X$ be a metric space. Then for any compact subset $K$ with $\mathcal{P}_{0}^{g}(K)<\infty$, we have

$$
\left(g_{*}(1-0)\right)^{2} \mathcal{P}_{0}^{g}(K) \leq \mathcal{P}^{g}(K) \leq \mathcal{P}_{0}^{g}(K) .
$$


Proof. It suffices to prove the first inequality of (14). Let $\varepsilon>0$. By the definition of $\mathcal{P}^{g}$, there exists a countable family $\left\{F_{i}\right\}_{i \geq 1}$ of sets such that $\bigcup_{i \geq 1} F_{i}=K$ and

$$
\mathcal{P}^{g}(K)+\varepsilon \geq \sum_{i=1}^{\infty} \mathcal{P}_{0}^{g}\left(F_{i}\right) .
$$

From Theorem 1 we see that for every $i$ there is an open set $U_{i}$ such that $U_{i} \supset \operatorname{cl}\left(F_{i}\right)$ and

$$
\mathcal{P}_{0}^{g}\left(\operatorname{cl}\left(F_{i}\right)\right) \geq g_{*}(1-0)\left(\mathcal{P}_{0}^{g}\left(U_{i}\right)-\varepsilon / 2^{i}\right) .
$$

Since $K$ is compact and $\left\{U_{i}\right\}$ is an open covering of $K$, we may choose a finite subcovering, say $K \subset \bigcup_{i=1}^{N} U_{i}$. From the finite subadditivity of $\mathcal{P}_{0}^{g}$ and the inequalities (13) and (15), we get

$$
\begin{aligned}
\mathcal{P}^{g}(K)+\varepsilon & \geq \sum_{i=1}^{\infty} \mathcal{P}_{0}^{g}\left(F_{i}\right) \geq g_{*}(1-0) \sum_{i=1}^{\infty} \mathcal{P}_{0}^{g}\left(\operatorname{cl}\left(F_{i}\right)\right) \\
& \geq\left(g_{*}(1-0)\right)^{2}\left(\sum_{i=1}^{\infty} \mathcal{P}_{0}^{g}\left(U_{i}\right)-\varepsilon\right) \\
& \geq\left(g_{*}(1-0)\right)^{2}\left(\sum_{i=1}^{N} \mathcal{P}_{0}^{g}\left(U_{i}\right)-\varepsilon\right) \\
& \geq\left(g_{*}(1-0)\right)^{2}\left(\mathcal{P}_{0}^{g}(K)-\varepsilon\right) .
\end{aligned}
$$

Letting $\varepsilon \rightarrow 0$, we finally obtain $\mathcal{P}^{g}(K) \geq\left(g_{*}(1-0)\right)^{2} \mathcal{P}_{0}^{g}(K)$.

Lemma 3 implies immediately

Corollary 1. For any compact set $K \subset X$ with $\mathcal{P}_{0}^{g}(K)<\infty$, we have:

(a) $0<\mathcal{P}^{g}(K)<\infty \Leftrightarrow 0<\mathcal{P}_{0}^{g}(K)<\infty$;

(b) $g_{*}(1-0)=1 \Rightarrow \mathcal{P}^{g}(K)=\mathcal{P}_{0}^{g}(K)$.

REMARK 1. It is known that a countable compact subset $E$ of $\mathbb{R}^{d}$ may have strictly positive upper box-counting dimension. However, from the result above we see that either $\mathcal{P}_{0}^{g}(E)=0$ or $\mathcal{P}_{0}^{g}(E)=\infty$ for every doubling gauge $g$.

Lemma 4. Let $X$ be a complete separable metric space with $\mathcal{P}^{g}(X)<\infty$ and let $B$ be a Borel subset of $X$. Then for any $\varepsilon>0$, there is a compact set $F \subset B$ with $\mathcal{P}_{0}^{g}(F)<\infty$ such that

$$
\mathcal{P}^{g}(F) \geq \mathcal{P}^{g}(B)-\varepsilon .
$$

Proof. Since $\mathcal{P}^{g}$ is a finite Borel measure on a complete separable metric space $X, \mathcal{P}^{g}$ is inner regular, thus it suffices to prove the statement under the assumption that $B$ is compact. By the definition of $\mathcal{P}^{g}$, the condition 
$\mathcal{P}^{g}(X)<\infty$ implies that there is a family $\left\{F_{i}\right\}$ of subsets with $B=\bigcup_{i=1}^{\infty} F_{i}$ such that

$$
\sum_{i=1}^{\infty} \mathcal{P}_{0}^{g}\left(F_{i}\right)<\infty
$$

Since $g$ is doubling we have $g_{*}(1-0)>0$. By Lemma 2, we may assume that all $F_{i}$ are compact. Let $\varepsilon>0$ and choose a positive integer $m$ such that

$$
\sum_{i=m+1}^{\infty} \mathcal{P}_{0}^{g}\left(F_{i}\right)<\varepsilon
$$

Take $F=\bigcup_{i=1}^{m} F_{i}$. In view of (17) and (18), we see that $F$ is a compact subset of $B$ such that

$$
\mathcal{P}_{0}^{g}(F) \leq \sum_{i=1}^{m} \mathcal{P}_{0}^{g}\left(F_{i}\right)<\infty
$$

and

$$
\mathcal{P}^{g}(B)-\mathcal{P}^{g}(F) \leq \mathcal{P}^{g}\left(\bigcup_{i=m+1}^{\infty} F_{i}\right) \leq \sum_{i=m+1}^{\infty} \mathcal{P}^{g}\left(F_{i}\right) \leq \sum_{i=m+1}^{\infty} \mathcal{P}_{0}^{g}\left(F_{i}\right)<\varepsilon
$$

THEOREM 2. Let $X$ be a complete separable metric space. Then for any Borel set $B \subset X$ we have

$$
\left(g_{*}(1-0)\right)^{2} \sup \mathcal{P}_{0}^{g}(F) \leq \mathcal{P}^{g}(B) \leq \sup \mathcal{P}_{0}^{g}(F)
$$

where the supremum is taken over all compact subsets of $B$ with $\mathcal{P}_{0}^{g}(F)<\infty$.

Proof. The first inequality in (19) follows directly from Lemma 3, and the second can be obtained immediately from Lemma 4 if $\mathcal{P}^{g}(X)<\infty$. To complete the proof, it suffices to prove the second inequality in the case $\mathcal{P}^{g}(X)=\infty$. Without loss of generality, assume $\mathcal{P}^{g}(B)>0$. Let $\beta \in\left(0, \mathcal{P}^{g}(B)\right)$. From the existence theorem of H. Joyce and D. Preiss [5], there is a compact subset $E \subset B$ such that $\beta \leq \mathcal{P}^{g}(E)<\infty$. Applying Lemma 4 to the set $E$, we get $\sup \mathcal{P}_{0}^{g}(F) \geq \beta$. Since $\beta \in\left(0, \mathcal{P}^{g}(B)\right)$ can be picked arbitrarily, we obtain $\sup \mathcal{P}_{0}^{g}(F) \geq \mathcal{P}^{g}(B)$ as required.

Theorem 2 immediately gives the following corollary.

Corollary 2. Let $B$ be a Borel subset of a complete separable space. Then:

(a) $\mathcal{P}^{g}(B)>0$ if and only if $B$ contains a compact subset $F$ such that $0<\mathcal{P}_{0}^{g}(F)<\infty$

(b) $\mathcal{P}^{g}(B)<\infty$ if and only if there is $\lambda \in[0, \infty)$ such that for any compact subset $F \subset B$, either $\mathcal{P}_{0}^{g}(F) \leq \lambda$ or $\mathcal{P}_{0}^{g}(F)=\infty$;

(c) $g_{*}(1-0)=1 \Rightarrow \mathcal{P}^{g}(B)=\sup \mathcal{P}_{0}^{g}(F)$. 
Using the above results, we are going to prove that for every doubling gauge, there is a compact metric space which has finite positive packing measure with respect to the gauge. This result is analogous to the existence theorem proved by A. Dvoretzky for Hausdorff measures [2].

Lemma 5. Suppose that $X$ is a separable metric space. Then for any subset $K$ of $X$,

$$
\mathcal{H}^{g}(K) \leq \mathcal{P}^{g}(K)
$$

where $\mathcal{H}^{g}(K)$ denotes the Hausdorff measure of $K$ with respect to the gauge $g$.

Proof. The proof is completely analogous to the proof of the case $g(t)=$ $t^{s}$ and $X=\mathbb{R}^{n}$ which can be found in P. Mattila [6].

TheORem 3. Let $g$ be any doubling gauge. Then there is a compact metric space $X$ such that

$$
0<\mathcal{P}^{g}(X)<\infty
$$

Proof. There is a compact metric space $\Omega$ such that $H^{g}(\Omega)>0$ (see Theorem 36 in [7]), so $\mathcal{P}^{g}(\Omega)>0$ by Lemma 5 . By Corollary $2($ a), there is a compact subset $X \subset \Omega$ such that $0<\mathcal{P}_{0}^{g}(X)<\infty$. Then by Corollary 1(a), we finally get $0<\mathcal{P}^{g}(X)<\infty$.

4. An example. Theorem 2 states that for any Borel set $G$ in a complete separable metric space,

$$
g_{*}(1-0)=1 \Rightarrow \mathcal{P}^{g}(G)=\sup \mathcal{P}_{0}^{g}(F),
$$

where the supremum is taken over all compact subsets contained in $G$ with $\mathcal{P}_{0}^{g}(F)<\infty$. We will show by giving a counterexample that the implication cannot be inverted, even if both $\mathcal{P}^{g}(G)$ and $\sup \mathcal{P}_{0}^{g}(F)$ are finite positive.

Let $G=[0,1]$ and $g:[0, \infty) \rightarrow[0, \infty)$ be defined by

$$
g(t)= \begin{cases}2^{-n} & \text { if } 2^{-n} \leq t \leq\left(1-2^{-n}\right) 2^{-n+1}, n \in \mathbb{N}, \\ 2^{n-1} t+2^{-n+1}-1 & \text { if }\left(1-2^{-n}\right) 2^{-n+1} \leq t \leq 2^{-n+1}, n \in \mathbb{N} .\end{cases}
$$

It is easy to verify that

$$
\frac{1}{2}=\liminf _{t \rightarrow 0} \frac{g(t)}{t} \leq \limsup _{t \rightarrow 0} \frac{g(t)}{t}=1,
$$

and thus $g$ is a doubling gauge. We are going to prove that

$$
\mathcal{P}^{g}(G)=\mathcal{P}_{0}^{g}(G)=1, \quad \text { but } \quad g_{*}(1-0)=1 / 2 .
$$

Let $x_{k}=1-2^{-k}$ and $t_{n}=2^{-n}, k, n \in \mathbb{N}$. Fix $k \in \mathbb{N}$. By the construction of $g$ we have

$$
\begin{aligned}
\liminf _{t \rightarrow 0} \frac{g\left(t x_{k}\right)}{g(t)} & \leq \liminf _{n \rightarrow \infty} \frac{g\left(t_{n} x_{k}\right)}{g\left(t_{n}\right)} \leq \liminf _{n \rightarrow \infty} \frac{g\left(\left(1-2^{-n-1}\right) 2^{-n}\right)}{g\left(2^{-n}\right)} \\
& =\lim _{n \rightarrow \infty} \frac{2^{-n-1}}{2^{-n}}=\frac{1}{2}
\end{aligned}
$$


which yields $g_{*}\left(x_{k}\right) \leq 1 / 2$. Letting $k \rightarrow \infty$, we get $g_{*}(1-0) \leq 1 / 2$ since $g_{*}$ is non-decreasing. On the other hand, by the inequality (21), we have

$$
\liminf _{t \rightarrow 0} \frac{g(t x)}{g(t)} \geq \frac{x}{2}
$$

for all $x>0$, and thus $g_{*}(1-0) \geq 1 / 2$. We have thus proved the last equality of $(22)$.

Notice that $(21)$ implies that $\frac{1}{2} \mathcal{P}_{0}^{1}(F) \leq \mathcal{P}_{0}^{g}(F) \leq \mathcal{P}_{0}^{1}(F)$ for any Borel set $F$ on the real line, thus

$$
\frac{1}{2} \mathcal{P}^{1} \leq \mathcal{P}^{g} \leq \mathcal{P}^{1}
$$

for any Borel sets. Note that $\mathcal{P}^{1}$ is equal to the 1-dimensional Lebesgue measure and $\mathcal{P}^{g}$ is translation invariant and locally finite, so there is a number $c>0$ such that $\mathcal{P}^{g}=c \mathcal{P}^{1}$. In addition, analogously to the lower density theorem for the $s$-dimensional packing measure (see Theorem 6.10 of $[6]$ ), we have

$$
\liminf _{r \rightarrow 0} \frac{\mathcal{P}^{g}(G \cap B(x, r))}{g(2 r)}=1
$$

for $\mathcal{P}^{g}$-almost all $x \in G$. Invoking the Lebesgue density theorem we get

$$
c=\limsup _{r \rightarrow 0} \frac{g(t)}{t}=1,
$$

and so $\mathcal{P}^{g}=\mathcal{P}^{1}$. Since $\mathcal{P}_{0}^{1}(G)=\mathcal{P}^{1}(G)=1$ we then get

$$
1=\mathcal{P}^{1}(G)=\mathcal{P}^{g}(G) \leq \mathcal{P}_{0}^{g}(G) \leq \mathcal{P}_{0}^{1}(G)=1,
$$

which yields the first two equalities in (22).

\section{References}

[1] M. Csörnyei, An example illustrating $P^{g}(K) \neq P_{0}^{g}(K)$ for sets of finite pre-measure, Real Anal. Exchange 27 (2001/02), 65-70.

[2] A. Dvoretzky, A note on Hausdorff dimension functions, Proc. Cambridge Philos. Soc. 44 (1948), 13-16.

[3] K. J. Falconer, Fractal Geometry: Mathematical Foundations and Applications, Wiley, 1990.

[4] D. J. Feng, S. Hua and Z. Y. Wen, Some relations between packing pre-measure and packing measure, Bull. London Math. Soc. 31 (1999), 665-670.

[5] H. Joyce and D. Preiss, On the existence of subsets of finite positive packing measure, Mathematika 42 (1995), 15-24.

[6] P. Mattila, Geometry of Sets and Measures in Euclidean Spaces, Cambridge Univ. Press, 1995.

[7] C. Rogers, Hausdorff Measures, Cambridge Univ. Press, 1970, 1998.

[8] S. J. Taylor and C. Tricot, Packing measure and its evaluation for Brownian paths, Trans. Amer. Math. Soc. 288 (1985), 679-699. 
[9] C. Tricot, Two definitions of fractional dimension, Math. Proc. Cambridge Philos. Soc. 91 (1982), 57-74.

Department of Mathematics

Department of Mathematics

Hubei University

Hubei, 430062, P.R. China

Tsinghua University

E-mail: sywen_65@163.com Beijing, 100084, P.R. China

E-mail: wenzy@tsinghua.edu.cn

Received September 22, 2003

Revised version March 15, 2004 